\title{
Trapianto renale da donatore vivente: il giovane nefrologo lo consiglia al giovane paziente nefropatico
}

\author{
Chiara Caletti
}

Sezione di Nefrologia, Dipartimento di Medicina, Università di Verona-Azienda Ospedaliera Universitaria Integrata, Verona

\begin{abstract}
LIVING-DONOR RENAL TRANSPLANTATION: THE RECOMMENDATIONS OF A YOUNG NEPHROLOGISTS TO A YOUNG NEPHROPATIC PATIENT Abstract. Renal transplantation is the gold-standard renal replacement therapy for patients affected by advanced renal failure; specifically, the living-donor transplantation program represents the best therapeutic option for young patients. It is well known that avoiding dialysis can significantly reduce comorbidities and increase patient survival. However, today in Italy the living-donor transplantation is still less common than the cadaveric one. Possible reasons may be related to the absence of a well-standardized pre-dialysis clinical followup and to the insufficient information provided to patients about the ongoing Italian transplant programs (including pre-emptive living transplantation).

Therefore, I strongly believe that, in order to improve this fundamental program, a social and a psychological support should be introduced to improve the timing and characteristics of the clinical follow-up of patients with end-stage renal failure and for the optimization of the pre-transplant clinical evaluations, thus reducing time and costs.
\end{abstract}

Finally, I think that new regional or national projects aimed at increasing living donations should be encouraged.

Key words: Kidney Transplant, Living donors, Pre-dialysis

Conflict of interest: None.

Financial support: None.

Accettato: 10 Febbraio 2014

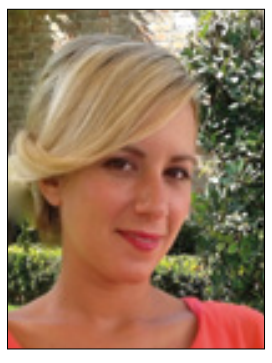

Chiara Caletti

\section{L'origine della passione trapiantologica}

La nefrologia è una disciplina varia e interessante, costituita da un'enorme multidisciplinarietà, ma il trapianto renale è indubbiamente uno degli argomenti che, durante il mio percorso formativo, ho trovato particolarmente affascinante. La mia formazione nefrologica è iniziata all'Università degli Studi del Piemonte Orientale, durante il corso di laurea, e prosegue tuttora presso la Scuola di Specializzazione in Nefrologia all'Università di Verona; in entrambi i casi vi è una fervente attività di follow-up pre- e post-trapianto. Tra le varie esperienze in questo affascinante mondo, ricordo ancora il primo contatto con una coppia candidata al trapianto da donatore vivente: figlio affetto da insufficienza renale ormai giunta in fase avanzata e mamma fermamente convinta di fare il possibile per aiutarlo. Sono rimasta immediatamente affascinata dal gesto di amore e altruismo di quella madre, anche se, chiaramente, il trapianto da vivente non deve essere basato solo su un rapporto di sentimento donatore-ricevente.

Con il progredire dei miei studi, sono diventata sempre più consapevole della frequenza e della complessità della patologia nefrologica per cui il trapianto renale risulta essere una delle terapie ottimali.

Allo stato attuale, in Italia, il trapianto renale da donatore cadavere è la strada più praticata, soprattutto per questioni organizzative di ogni singolo centro e, purtroppo, anche per la mancanza di un adeguato counseling al paziente circa la possibilità del trapianto da donatore vivente. Io sono, infatti, fermamente convinta che sia fondamentale incentivare il trapianto da donatore vivente, soprattutto nel paziente giovane. Il trapianto da vivente è, infatti, il gold standard terapeutico per il paziente under 40 , in cui l'aspettativa di vita è maggiore e in cui la disponibilità di organi di pari età è limitata; si rischia, infatti, che, per i pazienti appartenenti a questa fascia di età, il tempo di attesa in lista attiva sia decisamente più lungo, con il rischio del sopraggiungere delle complicanze cardiovascolari e osteometaboliche del paziente uremico (1). Il trapianto renale da donatore vivente presenta, inoltre, innumerevoli vantaggi, in termini sia chirurgici (pianificazione dell'intervento, riduzione dei tempi di ischemia fredda) che 
clinici, in particolare per la possibilità di eseguire l'intervento prima dell'inizio del trattamento dialitico (2).

\section{Approccio metodologico}

Il paziente nefropatico più frequentemente giunge alle fasi avanzate dell'insufficienza renale dopo un follow-up nefrologico di durata variabile. È fondamentale che questo percorso sia condotto in un ambiente idoneo e protetto come un ambulatorio di predialisi organizzato. È proprio in questi mesi o anni che il paziente e i familiari devono essere accompagnati nella scelta della tecnica sostitutiva della funzione renale e in cui il nefrologo può adeguatamente informare circa la donazione da vivente, soprattutto circa la possibilità del pre-emptive. A mio parere, risulta, quindi, indispensabile che ogni reparto di Nefrologia e Dialisi sia dotato di un ambulatorio di predialisi, costituito da personale medico e infermieristico adeguatamente formato sul tema del trapianto da vivente; di indubbia importanza è la figura dello psicologo, che deve accompagnare il paziente sia nella scelta di intraprendere questa strada che nella gestione delle delicate dinamiche tra donatore e ricevente (3).

Utile potrebbe essere l'utilizzo di manuali informativi, sia cartacei che informatizzati, ma può essere utile anche la creazione di incontri e conferenze in cui il personale sanitario possa rispondere a dubbi e perplessità dei pazienti e dei familiari, coinvolgendo nell'organizzazione altri pazienti che hanno già affrontato un percorso analogo e che possono, pertanto, portare la propria esperienza personale.

Una volta selezionata la "coppia", è importante che ricevente e donatore siano sottoposti a uno studio clinico rapido ed efficace, in modo da velocizzare il processo e da evitare l'inizio della terapia sostitutiva.

Un altro punto importante è il follow-up post-trapianto del donatore. Non bisogna mai dimenticare che il donatore è una persona sana che volontariamente si sottopone a un rischio clinico e chirurgico solo ed esclusivamente per la volontà di donare. È, quindi, fondamentale che i rischi chirurgici e nell'immediato post-operatorio siano ridotti al minimo ma anche che il donatore sia seguito con un follow-up post-donazione. Ma chi si prende carico di questo? Io penso che sia importante coinvolgere $\mathrm{i}$ Medici di Medicina Generale nella gestione del donatore, che dovrà essere seguito con un follow-up clinico periodico, inserendo questa attività nella sua pratica clinica quotidiana (4).

\section{Conclusioni}

Il trapianto da donatore vivente è un'ottima alterativa al trapianto da cadavere, soprattutto nel paziente giovane che non ha ancora iniziato la dialisi, per il quale presenta innumerevoli vantaggi. È, pertanto, dovere di ogni nefrologo informare il paziente di questa possibilità e, dove praticabile, incentivare il paziente e i familiari alla donazione. Questo discorso è più utile per i giovani nefrologi come me che non dovrebbero considerare il trapianto renale un argomento di nicchia ma, al contrario, come la modalità di sostituzione della funzione renale di prima scelta.

La Società Italiana di Nefrologia può sicuramente dare un contributo in tal senso, promuovendo corsi di formazione su questa tematica, indirizzati soprattutto ai giovani nefrologi che si troveranno, in futuro, a gestire una popolazione di nefropatici sempre più numerosa e complessa.

\section{Riassunto}

Il trapianto renale è sicuramente la migliore tecnica di sostituzione della funzione renale nei pazienti con insufficienza renale cronica avanzata e, in particolare, il trapianto renale da donatore vivente rappresenta un'ottima opzione terapeutica per i pazienti giovani. È, infatti, ampiamente dimostrato che evitare il trattamento dialitico riduce sensibilmente le comorbidità e migliora la sopravvivenza. Allo stato attuale, il trapianto da donatore vivente occupa un posto di secondo piano rispetto al trapianto da donatore cadavere. I motivi di tale discrepanza sono da attribuire soprattutto all'assenza di una rete di ambulatori predialisi organizzata ed efficiente e alla mancanza di un'esaustiva informazione al paziente circa il programma di trapianto in Italia (in particolar modo, il trapianto pre-emptive).

Io credo, quindi, che sia necessario promuovere un approccio metodologico, in cui il paziente sia gestito non solo dal punto di vista clinico, ma anche da quello socio-assistenziale e psicologico, in modo da ottimizzare la valutazione clinica pre-trapianto, riducendo tempi e costi.

Penso che sia fondamentale la promozione di progetti regionali e nazionali volti a incrementare la donazione da vivente.

Parole chiave: Trapianto renale, Donatore vivente, Predialisi

Dichiarazione di conflitto di interessi: L'Autore dichiara di non avere conflitto di interesse.

Contributi economici agli Autori: L'Autore dichiara di non aver ricevuto sponsorizzazioni economiche per la preparazione dell'articolo.

\section{Indirizzo dell'Autore:}

Dr.ssa Chiara Caletti

Sezione di Nefrologia, Dipartimento di Medicina

Università di Verona

Piazzale Stefani 1

37100 Verona

caletti_chiara@yahoo.it

\section{Bibliografia}

1. The European Renal Best Practice (ERBP) transplantation guideline development group. ERBP guideline on the management and evaluation of the kidney donor and recipient. Nephrol Dial Transplant 2013; 28 (Suppl. 2).

2. Davis CL. Preemptive transplantation and the transplant first initiative. Curr Opin Nephrol Hypertens 2010; 19 (6): 592-7.

3. Schena A, Maiorano AM, Zaza G. Selection and follow-up of living donor kidney transplant recipients. G Ital Nefrol 2009; 26 (4): 468-77.

4. Delanaye P, Weekers L, Dubois BE, et al. Outcome of the living kidney donor. Nephrol Dial Transplant 2012; 27 (1): 41-50. 